\title{
EXPANSION OF THE GREEN'S FUNCTION IN A CYLINDRICAL COORDINATE SYSTEM
}

\author{
BY \\ WAN-XIAN WANG \\ Space Astronomy Laboratory, Institute for Space Science and Technology, Gainesville, Florida
}

Abstract. The expansions of the Green's function $e^{i k\left|\mathbf{r}-\mathbf{r}^{\prime}\right|} /\left|\mathbf{r}-\mathbf{r}^{\prime}\right|$ in spherical and spheroidal coordinate systems were studied by some researchers; however, because of its complexity this kind of expansion has not been performed in a cylindrical coordinate system. With the aid of the expressions of the Bessel functions $J_{-1 / 2}$ and $J_{1 / 2}$ and of the transformation from these functions to the spherical Bessel functions, the author has analytically derived the expansion of the Green's function in the cylindrical coordinate system.

1. Introduction. The expansion of the Green's function in a spherical coordinate system can be written in the form [1,2]

$$
\begin{aligned}
\frac{e^{i k\left|\mathbf{r}-\mathbf{r}^{\prime}\right|}}{\left|\mathbf{r}-\mathbf{r}^{\prime}\right|}= & i k \sum_{n=0}^{\infty}(2 n+1) j_{n}(k r) h_{n}^{(1)}\left(k r^{\prime}\right) \\
& \times\left\{P_{n}(\cos \theta) P_{n}\left(\cos \theta^{\prime}\right)\right. \\
& \left.+2 \sum_{m=1}^{n} \frac{(n-m) !}{(n+m) !} P_{n}^{m}(\cos \theta) P_{n}^{m}\left(\cos \theta^{\prime}\right) \cos m\left(\phi-\phi^{\prime}\right)\right\}
\end{aligned}
$$

where $(r, \theta, \phi)$ and $\left(r^{\prime}, \theta^{\prime}, \phi^{\prime}\right)$ are the spherical coordinates for the field and source points, $k$ is the wave number, $j_{n}$ and $h_{n}^{(1)}$ are the spherical Bessel and Hankel functions of the first kind, respectively, $P_{n}$ and $P_{n}^{m}$ are the Legendre and the associated Legendre functions of the first kind, respectively. In the case for $r>r^{\prime}$, just switch $j_{n}$ and $h_{n}^{(1)}$.

The expansion of the Green's function in a spheroidal coordinate system can be expressed as [3]

$$
\begin{array}{r}
\frac{e^{i k\left|\mathbf{r}-\mathbf{r}^{\prime}\right|}}{\left|\mathbf{r}-\mathbf{r}^{\prime}\right|}=2 i k \sum_{m=0}^{\infty} \sum_{n=m}^{\infty} \frac{2-\delta_{0 m}}{N_{m n}} R_{m n}^{(1)}(c, \xi) R_{m n}^{(3)}\left(c, \xi^{\prime}\right) S_{m n}(c, \eta) S_{m n}\left(c, \eta^{\prime}\right) \cos m\left(\phi-\phi^{\prime}\right) \\
\text { for } \xi<\xi^{\prime}, \quad(2)
\end{array}
$$

Received July 18,1989.

(C)1990 Brown University 
where $(\xi, \eta, \phi)$ and $\left(\xi^{\prime}, \eta^{\prime}, \phi^{\prime}\right)$ are respectively the spheroidal coordinates for the field and source points, $R_{m n}^{(1)}$ and $R_{m n}^{(3)}$ are respectively the spheroidal radial functions of the first and third kinds, $S_{m n}$ are the spheroidal angular functions, $\delta_{0 m}$ are the Kronecker delta functions, the focal parameter $c=k \ell$ in which $\ell$ is the semi-focal distance in the spheroidal coordinate system and $N_{m n}$ is the normalization factor defined in Flammer's book [3]. In the case $\xi>\xi^{\prime}$, just switch $R_{m n}^{(1)}$ and $R_{m n}^{(3)}$.

2. Expansion of the Green's function in a cylindrical coordinate system. The Green's function in a cylindrical coordinate system can be obtained from the following derivative:

$$
\frac{e^{i k\left|\mathbf{r}-\mathbf{r}^{\prime}\right|}}{\left|\mathbf{r}-\mathbf{r}^{\prime}\right|}=\frac{1}{\left[i k\left(z-z^{\prime}\right)\right]} \frac{d}{d\left(z-z^{\prime}\right)}\left[e^{i k\left|\mathbf{r}-\mathbf{r}^{\prime}\right|}\right],
$$

where $\left|\mathbf{r}-\mathbf{r}^{\prime}\right|=\left[\left(z-z^{\prime}\right)^{2}+r^{2}+r^{\prime 2}-2 r r^{\prime} \cos \left(\phi-\phi^{\prime}\right)\right]^{1 / 2}$, in which $(r, \phi, z)$ and $\left(r^{\prime}, \phi^{\prime}, z^{\prime}\right)$ are the cylindrical coordinates for the field and source points.

In order to simplify the derivation, we designate

$$
R=\left[\left(z-z^{\prime}\right)^{2}+r^{2}+r^{\prime 2}\right]^{1 / 2} \text {. }
$$

From the Lommel's expansions of $(Z+h)^{ \pm \nu / 2} J_{\nu}(\sqrt{Z+h})$ [4: Sec. 5.22], we have

$$
\begin{gathered}
(Z+h)^{-\nu / 2} J_{\nu}(\sqrt{Z+h})=\sum_{m=0}^{\infty} \frac{\left(-\frac{1}{2} h\right)^{m}}{m !} Z^{-(\nu+m) / 2} J_{\nu+m}(\sqrt{Z}), \\
(Z+h)^{\nu / 2} J_{\nu}(\sqrt{Z+h})=\sum_{m=0}^{\infty} \frac{\left(\frac{1}{2} h\right)^{m}}{m !} Z^{(\nu-m) / 2} J_{\nu-m}(\sqrt{Z}) \text { for } Z+h \neq 0,
\end{gathered}
$$

where $J_{\nu}$ is the Bessel function of order $\nu$.

Substituting $Z=k^{2} R^{2}$ and $h=-2 k^{2} r r^{\prime} \cos \left(\phi-\phi^{\prime}\right)$, and then setting $\nu=-\frac{1}{2}$ and $\nu=\frac{1}{2}$ in Eqs. (5.1) and (5.2), respectively, we obtain

$$
\begin{gathered}
k^{1 / 2}\left[R^{2}-2 r r^{\prime} \cos \left(\phi-\phi^{\prime}\right)\right]^{1 / 4} J_{-1 / 2}\left(k \sqrt{R^{2}-2 r r^{\prime} \cos \left(\phi-\phi^{\prime}\right)}\right) \\
=\sum_{m=0}^{\infty} \frac{\left[k^{2} r r^{\prime} \cos \left(\phi-\phi^{\prime}\right)\right]^{m}}{m !}(k R)^{(1-2 m) / 2} J_{-1 / 2+m}(k R), \\
k^{1 / 2}\left[R^{2}-2 r r^{\prime} \cos \left(\phi-\phi^{\prime}\right)\right]^{1 / 4} J_{1 / 2}\left(k \sqrt{R^{2}-2 r r^{\prime} \cos \left(\phi-\phi^{\prime}\right)}\right) \\
=\sum_{m=0}^{\infty} \frac{\left[-k^{2} r r^{\prime} \cos \left(\phi-\phi^{\prime}\right)\right]^{m}}{m !}(k R)^{(1-2 m) / 2} J_{1 / 2-m}(k R) .
\end{gathered}
$$

Expressions in Eqs. (6.1) and (6.2) may be regarded as the real and imaginary parts of $\sqrt{2 / \pi} e^{i k \sqrt{R^{2}-2 r r^{\prime} \cos \left(\phi-\phi^{\prime}\right)}}$. Thus, further simplifications for the combination of Eqs. (6.1) and (6.2) yield

$$
e^{i k \sqrt{R^{2}-2 r r^{\prime} \cos \left(\phi-\phi^{\prime}\right)}}=e^{i k R}+\sum_{m=1}^{\infty} \frac{\left(k^{2} r r^{\prime}\right)^{m} h_{m-1}^{(1)}(k R)}{m !(k R)^{m-1}} \cos ^{m}\left(\phi-\phi^{\prime}\right),
$$


where the following relations have been used:

$$
\begin{aligned}
\sqrt{\pi k R / 2}\left[J_{-1 / 2+m}(k R)+i(-1)^{m} J_{1 / 2-m}(k R)\right] & =(k R)\left[j_{m-1}(k R)+i(-1)^{m} j_{-m}(k R)\right] \\
& =(k R) h_{m-1}^{(1)}(k R) .
\end{aligned}
$$

By taking the derivative of Eq. (8) with respect to $\left(z-z^{\prime}\right)$ and using the recursion relations for the spherical Hankel functions of the first kind, the Green's function in the cylindrical coordinate system is expanded as follows:

$$
\frac{e^{i k\left|\mathbf{r}-\mathbf{r}^{\prime}\right|}}{\left|\mathbf{r}-\mathbf{r}^{\prime}\right|}=\frac{e^{i k R}}{R}+i k \sum_{m=1}^{\infty} \frac{\left(k^{2} r r^{\prime}\right)^{m} h_{m}^{(1)}(k R)}{m !(k R)^{m}} \cos ^{m}\left(\phi-\phi^{\prime}\right) \quad \text { except for } \mathbf{r}=\mathbf{r}^{\prime} .
$$

Acknowledgment. The author would like to express his appreciation of the referee's helpful suggestion.

\section{REFERENCES}

[1] J. D. Jackson, Classical Electrodynamics, John Wiley and Sons, New York, 1962

[2] T. S. M. Maclean, Principles of Antennas: Wire and Aperture, Cambridge University Press, 1986

[3] C. Flammer, Spheroidal Wave Functions, Stanford University Press, 1957

[4] G. N. Watson, A Treatise on the Theory of Bessel Functions, Cambridge University Press, 1962 